\title{
WEAKLY ALMOST PERIODIC FUNCTIONS AND FOURIER-STIELTJES TRANSFORMS
}

\author{
DONALD E. RAMIREZ ${ }^{1}$
}

Let $G$ be an infinite nondiscrete abelian group; $\Gamma$ the (noncompact) dual group of $G ; M(G)$ the algebra of bounded Borel measures on $G$; $M(G)^{\wedge}$ the algebra of Fourier-Stieltjes transforms; $M(G)^{\wedge-}$ the completion of $M(G)^{\wedge}$ in the 'sup-norm topology on $\Gamma$; and WAP $(\Gamma)$ the algebra of continuous bounded weakly almost periodic functions on $\Gamma$.

The object of this paper is to show the following theorem.

THEOREM. Let $\Gamma$ be an infinite noncompact abelian group. Then $M(G)^{\wedge-} \neq \mathrm{WAP}(\Gamma)$.

Proof. We consider first the case that $\Gamma$ is discrete. If $\Gamma$ is not of bounded order, then Rudin [4] using a deep trigonometric inequality has shown this result. A proof based on an elementary inequality may be found in [3].

Thus we may assume that $\Gamma$ is of bounded order. Let $Z(p)$ denote the finite cyclic group of $p$ elements of unimodular complex numbers; and $Z(p)^{\infty}$ the weak direct product of $Z(p)$ over a countable infinite index set. Thus there exists $p$ such that $Z(p)^{\infty}$ is a subgroup of $\Gamma$, $\left[1\right.$, p. 449]. We may assume that $\Gamma=Z(p)^{\infty}$.

There exists $\lambda_{n} \in M(\Gamma)$ such that $\left\|\lambda_{n}\right\|=1$ and $\left\|\lambda_{n}^{\wedge}\right\|_{\infty} \leqq 1 / n, n$ $=1,2, \cdots$. Let $S_{n}$ denote the supp $\lambda_{n}$. We may assume that the $S_{n}$ 's are finite sets and pairwise disjoint e.g. [2, Theorem 3.2]. Let $f_{0}$ be a (continuous) bounded function on $\Gamma$ such that $\int_{\Gamma} f_{0} d \lambda_{n}=\left\|\lambda_{n}\right\|=1$, $\left\|f_{0}\right\|_{\infty} \leqq 1$, and $\operatorname{supp} f_{0}=\mathrm{U}_{n=1}^{\infty} S_{n}$.

Let $g$ be a (continuous) bounded function on $\Gamma . g \in M(G)^{\wedge-}$ if and only if $\left\{\lambda_{n}\right\} \subset M(\Gamma),\left\|\lambda_{n}\right\| \leqq 1$, and $\lambda_{n}^{\wedge}(x) \stackrel{n}{\rightarrow} 0$ for all $x \in G$ implies $\int_{\Gamma} g d \lambda_{n} \stackrel{n}{\rightarrow} 0$, [2, Theorem 1.9]. Thus $f_{0} \notin M(G)^{\wedge}$-. It remains to show that we may pick $f_{0}$ such that $f_{0} \in \mathrm{WAP}(\Gamma)$.

Let $S=\cup_{n=1}^{\infty} S_{n}$. It is enough to construct $S$ such that $S \cap(S+y)$ is finite for every $y \neq 0$ since Rudin [4] has shown that any continuous

Received by the editors June 1,1967 .

1 The author is a research associate of the Office of Naval Research, contract number N00014-66-C0269.

The author wishes to thank the referee for being extremely prompt and for pointing out that the general case follows from the discrete case via the structure theorem for locally compact abelian groups. 
bounded function $f$ with $\operatorname{supp} f=S$ would then be weakly almost periodic. A proof using quasi-uniform convergence may be found in $[3]$.

Let $H \subset \Gamma$ be a finite set. Let $\alpha(H)[\beta(H)]$ denote the last [first] coordinate such that all elements of $H$ are 1 for coordinates $<\alpha(H)[>\beta(H)]$. Let the $S_{n}$ 's be constructed such that $\alpha\left(S_{1}\right)<\beta\left(S_{1}\right)$ $<\alpha\left(S_{2}\right)<\beta\left(S_{2}\right) \cdots<\alpha\left(S_{n}\right)<\beta\left(S_{n}\right)$. Thus $f_{0} \in \mathrm{WAP}(\Gamma)$.

Now let $\Gamma$ be any infinite noncompact abelian group. If $\Gamma$ contains a copy of $R^{n}$ then [4] applies. If not, then the structure theorem for locally compact abelian groups $[1$, p. 389] implies that $\Gamma$ contains a compact open subgroup $\Lambda . \Gamma / \Lambda$ is infinite and discrete. Let $f$ be the function on $\Gamma / \Lambda$ given by the preceding case. Finally, extend $f$ canonically to $\Gamma$. That $f \in M(G)^{\wedge-}$ follows from the characterization of $M(G)^{\wedge-},[2$, Theorem 1.9].

\section{BIBLIOGRAPHY}

1. Edwin Hewitt and Kenneth Ross, Abstract harmonic analysis. I, Academic Press, New York, 1963.

2. Donald Ramirez, Uniform approximation by Fourier-Stieltjes transforms, Proc. Cambridge Philos. Soc. 64 (1968), 323-333.

3. - Uniform approximation by Fourier-Stieltjes coefficients, Proc. Cambridge Philos. Soc. 64 (1968), 615-624.

4. Walter Rudin, Weak almost periodic functions and Fourier-Stieltjes transforms, Duke Math. J. 26 (1959), 215-220.

UNIVERSITY OF WASHINGTON AND UNIVERSITY OF VIRGINIA 